\title{
Zur Nichtfällbarkeit des Magnesiums durch Ammoniak bei Gegenwart von Ammonsalzen.
}

\author{
Von \\ F. P. Treadwell.
}

Dals Magnesium durch Ammoniak bei Gegenwart von Ammonsalzen nicht gefällt wird, wurde bis vor wenigen Jahren durch die Annahme von komplexen Salzen von der Zusammensetzung:

$$
\left[\mathrm{MgCl}_{3}\right] \mathrm{NH}_{4} \text {, oder }\left[\mathrm{MgCl}_{4}\right]\left(\mathrm{NH}_{4}\right)_{2} \text {, erklärt. }
$$

Beide Salze sind im festen Zustand isoliert, aber nicht als Komplex-, sondern als Doppelsalze beschrieben worden.

Wenn nun eines dieser beiden Salze die Ursache der Nichtfällbarkeit des Magnesiums ist, so müfste beim Fällen einer neutralen Magnesiumchloridlösung mit überschüfsigem Ammoniak die Reaktion verschieden verlaufen, je nachdem das Salz mit einem oder mit zwei $\mathrm{NH}_{4}$ entsteht.

Bildet sich also das Salz mit einem $\mathrm{NH}_{4}$, so würde die Reaktion nach dem Schema:

$$
3 \mathrm{MgCl}_{2}+2 \mathrm{NH}_{4} \mathrm{OH}=2\left[\mathrm{MgCl}_{3}\right] \mathrm{NH}_{4}+\mathrm{Mg}(\mathrm{OH})_{2}
$$

verlaufen, d. h. es würde genau ein Drittel des Magnesiums gefällt werden; bildet sich dagegen das $\mathrm{Salz}$ mit zwei $\mathrm{NH}_{4}$, so mürste genau die Hälfte des Magnesiums als Magnesiumhydroxyd abgeschieden werden:

$$
2 \mathrm{MgCl}_{2}+2 \mathrm{NH}_{4} \mathrm{OH}=\left[\mathrm{MgCl}_{4}\right]\left(\mathrm{NH}_{4}\right)_{2}+\mathrm{Mg}(\mathrm{OH})_{2} .
$$

In GMEwn-KRAUT ${ }^{1}$ findet sich folgende Angabe: „Überschülsiges Ammoniak fällt aus Chlormagnesiumlösung genau die Hälfte der

1 6. Aufl, Bd. II, S. 481. 
Magnesia, während dieses Doppelsalz gelöst bleibt." Hier ist aber das Doppelsalz mit einem $\mathrm{NH}_{4}$ gemeint. Mir erschien dies unwahrscheinlich und so liefs ich vor Jahren Versuche hierüber anstellen, welche wirklich ergaben, dafs sehr annähernd die Hälfte des Magnesiums durch Ammoniak gefällt wurde. Allerdings konnte schon damals konstatiert werden, dals, bei sehr grofser Konzentration des Ammoniaks, weit mehr als die Hälfte des Magnesiums abgeschieden wurde, und zwar nicht sofort, sondern allmählich; ein Umstand, der für den allmählichen Zerfall des Komplexsalzes zu sprechen schien.

Die Versuche, die ich damals anstellen liefs, wurden so ausgeführt: Eine neutrale Magnesiumchloridlösung von bekanntem Gehalt wurde in einem $100 \mathrm{ccm}$ Kolben mit einem Überschufs von Ammoniak versetzt, mit Wasser bis zur Marke aufgefüllt, nach dem Mischen sofort durch ein trockenes Filter filtriert und hierauf in einem aliquoten Teil des Filtrats das Magnesium bestimmt.

Loven ${ }^{1}$, der sehr ausgedehnte Versuche über die Fällung von Magnesium aus neutraler Lösung durch Ammoniak von der verschiedensten Konzentration angestellt hat, kommt zu dem Schlufs, dafs die Nichtfällbarkeit des Magnesiums durch Ammoniak nicht der Bildung komplexer Ionen, sondern lediglich der Zurückdrängung der Dissoziation des an und für sich sehr wenig dissoziierten Ammoniaks durch Ammonsalze zuzuschreiben sei.

Trotzdem die Lovisschen Zahlen fast überzeugend sind, so war es immerhin auffallend, dafs beim Fällen von Magnesiumchlorid mit einem Überschufs von Ammoniak fast genau die Hälfte des Magnesiums ausfällt. Ich glaubte daher immer noch, es müsse das komplexe Salz $\left[\mathrm{MgCl}_{4}\right]\left(\mathrm{NH}_{4}\right)_{2}$ entstehen, dieses aber nach und nach in Magnesiumchlorid und Chlorammonium zerfallen, wodurch erklärt würde, warum das Magnesium bei längerem Stehen sich immer mehr und mehr aus der Lösung als Magnesiumhydroxyd abscheidet. Tch liefs daher eine neue Versuchsreihe ausführen, um den zeitlichen Verlauf der Reaktion kennen zu lernen. Die Versuche wurden nicht nur bei Zimmertemperatur, sondern auch bei Null Grad ausgeführt. Ich hoffte durch Arbeiten bei niedriger Temperatur den Zerfall des Komplexsalzes sehr herabzumindern, wenn nicht ganz zu verhindern. Allein es zeigte sich, dafs auch uuter diesen Umständen die gefällte Magnesiummenge stets von der Konzentration der Hydroxylionen und von der Dauer des Versuchs abhängig ist.

1 J. M. Lovtix, Z. anorg. Chem. 11, 404, 
Ich lasse hier die Belege folgen.

Es wurden stets zwei Parallelversuche ausgeführt. Dieselbe Magnesiummenge wurde mit überschüssigem Ammoniak versetzt, auf $100 \mathrm{ccm}$ gebracht, die eine Probe sofort, die andere erst nach 24-stündigem Stehen filtriert und das Magnesium im Filtrat als $\mathrm{Mg}_{2} \mathrm{P}_{2} \mathrm{O}_{7}$ bestimmt.

\section{Serie I.}

$25 \mathrm{ccm}$ einer Magnesiumchloridlösung, enthaltend $0.3124 \mathrm{~g} \mathrm{Mg}$, wurden mit $75 \mathrm{ccm} 1 / 1$ norm. Ammoniaklösung gefällt.

$$
\text { Temperatur }=\mathrm{ca} .17^{\circ} \mathrm{C} .
$$

Angewandtes $\quad \mathrm{Mg}$ gefällt nach $\quad$ Von $100 \mathrm{Tl} . \mathrm{Mg} \quad \mathrm{Mg}$ gefällt Von $100 \mathrm{Tl} \mathrm{Mg}$ Magnesium sofortig. Filtration wurden gefällt nach $24 \mathrm{St}$. wurden gefällt
$0.3124 \mathrm{~g}$
$0.1521 \mathrm{~g}$
$48.7 \%$
$0.2641 \mathrm{~g}$
$84.6 \%$
Temperatur $=$ ca. $0^{\circ} \mathrm{C}$.
$0.3124 \mathrm{~g}$
$0.1368 \mathrm{~g}$
43.8
$0.2439 \mathrm{~g}$
$78.1 \%$

Serie II.

$25 \mathrm{ccm}$ Magnesiumchloridlösung, enthaltend $0.3124 \mathrm{~g} \mathrm{Mg}$, wurden mit $75 \mathrm{ccm}$ Ammoniak, wovon $1 \mathrm{ccm}=0.00608 \mathrm{~g} \mathrm{NH}_{3}$, versetzt. L's war also bei diesen Versuchen nur ein sehr geringer Überschufs von Ammoniak vorhanden. (Angewandt wurden $0.4560 \mathrm{~g} \mathrm{NH}_{3}$, während die berechnete Menge $0.4360 \mathrm{~g} \mathrm{NH}_{3}$ beträgt.)

$$
\text { Temperatur }=\text { ca. } 17^{\circ} \mathrm{C} .
$$

Angewandtes $\mathrm{Mg}$ gefällt nach Von $100 \mathrm{Tl} . \mathrm{Mg} \quad \mathrm{Mg}$ gefällt Von $100 \mathrm{Tl} . \mathrm{Mg}$ Magnesium sofortig. Filtration wurden gefällt nach $24 \mathrm{St}$. wurden gefällt

$$
\begin{array}{ccccc}
0.3124 \mathrm{~g} & 0.0589 \mathrm{~g} & 18.9 \% & 0.1357 \mathrm{~g} & 43.7 \% \\
\text { Temperatur }=0^{\circ} \mathrm{C} . \\
0.3124 \mathrm{~g} & 0.0524 \mathrm{~g} & 16.6 \% & 0.1094 \mathrm{~g} & 26.4 \%
\end{array}
$$

Aus den Versuchen von Serie I geht hervor, dals aus einer Magnesiumchloridlösung durch einen grofsen Überschufs von Ammoniak ungefähr die Hälfte des Magnesiums gefällt wird, wenn man sofort nach der Fällung filtriert. Dagegen wird nach 24 stündigem Stehen ca. $80 \%$ des Gesamtmagnesiums als Magnesiumbydroxyd abgeschieden, einerlei ob man bei höherer oder niedrigerer Temperatur arbeitet.

Die Versuche von Serie II, bei welcher nur ein geringer Überschufs an Ammoniak zur Anwendung kam, zeigen, dals nach sofortiger 
Filtration nicht einmal ein Fünftel, nach 24 Stunden bedeutend weniger als die Hälfte des vorhandenen Magnesiums gefällt wurde und zwar bei Null Grad bedeutend weniger als bei gewöhnlicher Temperatur. Auch nach mehr als 24 Stunden schied sich kein Magnesium mehr aus.

Um den zeitlichen Verlauf der Reaktion noch besser kennen zu lernen, wurde eine neue Versuchsreihe so angestellt, dals der entstandene Niederschlag nach $1 / 4,3 / 4$. 1, 2, 30, 60 Min., 6 Stunden abfiltriert und das in Filtrat befindliche Magnesium bestimmt wurde.

$25 \mathrm{ccm}$ einer Magnesiumchloridlösung, enthaltend $0.3115 \mathrm{~g} \mathrm{Mg}$, wurden mit $75 \mathrm{cem} 1 / 1$ normalem Ammoniak geschüttelt und filtriert wobei die ersten $5-10 \mathrm{ccm}$ stets vernachlässigt wurden.

Magnesium gefällt nach:

Angewandtes

Magnesium

$1 / 4$ Min.

$3 / 4$ Min.

1 Min.

$0.3115 \mathrm{~g} \quad 0.1604 \mathrm{~g}=51.5 \% \quad 0.1630 \mathrm{~g}=52.4 \% \quad 0.1631 \mathrm{~g}=52.4 \%$

2 Min.

30 Min.

1 Stunde

6 Stunden

$0.1755 \mathrm{~g}=56.3 \% 0.2518 \mathrm{~g}=80.5 \% \quad 0.2642 \mathrm{~g}=84.5 \% 0.2702 \mathrm{~g}=86.7 \%$

Aus diesen Versuchen ersieht man, dafs die Ausfällung des Magnesiums ungemein rasch erfolgt; schon nach $1 / 2$ Stunde sind bereits $80 \%$ des Gesamtmagnesiums gefällt. Alle diese Versuche bestätigen vollauf die Lovkss und sprechen entschieden gegen die Annahme von Komplexsalzen.

Um aber jeden $Z_{\text {weifel }}$ zu beseitigen, wurde eine Lösung von Magnesiumchlorid und Chlorammonium, im Verhältnis von $1 \mathrm{MgCl}_{2}$ $2 \mathrm{NH}_{4} \mathrm{Cl}$, hergestellt und das Molekulargewicht der gelösten Substanz auf kryoskopischen Wege bestimmt:

$$
\begin{gathered}
\text { Angewandte Substanz }=0.3113 \mathrm{~g}\left\{\begin{array}{l}
\mathrm{MgCl}_{2}=0.1466 \\
\mathrm{NH}_{4} \mathrm{Cl}=0.1647
\end{array}\right. \\
\text { Wasser }=20 \mathrm{~g} \\
\begin{aligned}
\Lambda & =0.9609
\end{aligned} \\
M=\frac{100 \cdot 18.5 \cdot 0.3113}{0.9609 \cdot 20}=29.97
\end{gathered}
$$

Das Molekulargewicht des Salzes $\left[\mathrm{MgCl}_{4}\right]\left(\mathrm{NH}_{4}\right)_{2}$ ist 202.32 . Wäre dieses Salz wirklich vorhanden, so mülste man für $M$ annährend $\frac{202.32}{3}=67.44$ finden. Wenn aber die Lösung nur eine Mischung 
von $\mathrm{MgCl}_{2}$ und $\mathrm{NH}_{4} \mathrm{Cl}$ enthält, so müfste sich riür $M=\frac{202.32}{7}=28.9$ ergeben, was sehr annährend zutrifft.

Zur Kontrolle wurde die Gefrierpunktserniedrigung einer Maynesiumchlorid- und einer Ammoniumchloridlösung, von derselben Magnesium- und Ammoniumkonzentration wie oben, ausgeführt:

$$
\begin{gathered}
\text { Angewandtes } \mathrm{MgCl}_{2}=0.1466 \mathrm{~g} \\
\text { Wasser }=20 \mathrm{~g} \\
\Delta=0.3956^{\circ} \\
M=\frac{18.5 \cdot 100 \cdot 0.1466}{0.3956 \cdot 20}=34.27
\end{gathered}
$$

statt der berechneten Zahl

$$
\begin{gathered}
\mathrm{MgCl}_{2} \\
\frac{3}{3}=\frac{95.26}{3}=31.75 . \\
\text { Angewandtes } \mathrm{NH}_{4} \mathrm{Cl}=0.1647 \mathrm{~g} \\
\text { Wasser }=20 \mathrm{~g} \\
\Delta=0.5511^{\circ} \mathrm{C} \\
M=\frac{18.5 \cdot 100 \cdot 0.1647}{0.5511 \cdot 20}=27.75
\end{gathered}
$$

statt der berechneten Zahl

$$
\frac{\mathrm{NH}_{4} \mathrm{Cl}}{2}=\frac{53.52}{2}=26.76
$$

Also fast vollständige Übereinstimmung.

Durch diese Versuche ist bewiesen, dafs das Magnesiumchlorid mit Ammonchlorid keine Komplexsalzbildung eingeht.

Znm Überflufs wurde die Gefrierpunktserniedrigung einer Manganammoniumchloridlösung bestimmt, weil anzunehmen war, dals sich das Mangan in bezug auf Komplexsalzbildung dem Magnesium ganz ähnlich verhalten würde.

$$
\begin{aligned}
& \text { Angewandte Substanz }=0.1782 \mathrm{~g}\left\{\begin{array}{l}
\mathrm{MnCl}_{2}=0.0968 \\
\mathrm{NH}_{4} \mathrm{Cl}=0.0824
\end{array}\right. \\
& \text { Wasser }=20 \mathrm{~g} \\
& \quad A=0.4797
\end{aligned}
$$




$$
M=\frac{18.5 \cdot 100 \cdot 0.1782}{0.4797 \cdot 20}=34.56
$$

statt der berechneten Zahl

$$
\frac{\left[\mathrm{MnCl}_{4}\right]\left(\mathrm{NH}_{4}\right)_{2}}{7}=\frac{232.96}{7}=\mathbf{3 3 . 2 8} \text {. }
$$

Also auch hier keine Komplexsalzbildung.

Durch diese Versuche wird die Lovensche Ansicht bestätigt, dals die Nichtfällbarkeit des Magnesiums (und ähnlicher Elemente) bei Gegenwart von Ammonsalzen, nicht durch Komplexsalzbildung bedingt wird, sondern lediglich durch Zurückdrängung der Dissoziation des Ammoniumbydroxyds durch das Ammoniumchlorid, entsprechend dem Massenwirkungsgesetz.

Meinen Assistenten Herrn Dr. F. Henz und Herrn E. WegeLin, die mir bei der Ausführung der oben beschriebenen Versuche behilflich waren, sage ich meinen besten Dank.

Zürich, analytische Abteilung des eidgen. Polytechnikums.

Bei der Redaktion eingegangen am 4. Oktober 1903. 